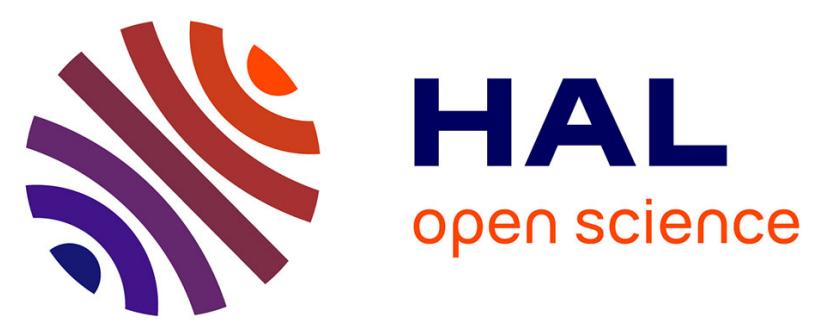

\title{
Effet du genre sur le choix et le rejet des activités physiques et sportives en Éducation Physique et Sportive: une approche additive et différentielle du modèle de l'androgynie
}

Paul Fontayne, Philippe Sarrazin, Jean-Pierre Famose

\section{To cite this version:}

Paul Fontayne, Philippe Sarrazin, Jean-Pierre Famose. Effet du genre sur le choix et le rejet des activités physiques et sportives en Éducation Physique et Sportive: une approche additive et différentielle du modèle de l'androgynie. Science et Motricité: revue scientifique de l'ACAPS / ACAPS, 2002, 45, pp.45-66. 10.3917/sm.045.0045 . hal-00387233

\section{HAL Id: hal-00387233 https://hal.science/hal-00387233}

Submitted on 25 May 2009

HAL is a multi-disciplinary open access archive for the deposit and dissemination of scientific research documents, whether they are published or not. The documents may come from teaching and research institutions in France or abroad, or from public or private research centers.
L'archive ouverte pluridisciplinaire HAL, est destinée au dépôt et à la diffusion de documents scientifiques de niveau recherche, publiés ou non, émanant des établissements d'enseignement et de recherche français ou étrangers, des laboratoires publics ou privés. 


\section{Effet du genre sur le choix et le rejet des activités physiques et sportives en Éducation Physique et Sportive : une approche additive et différentielle du modèle de l'androgynie.}

Paul Fontayne ${ }^{\star}$, Philippe Sarrazin ${ }^{\star *}$, et can-Pierre Famos*

\section{RÉSUMÉ}

Le domaine du sport et des activités physiques est traditionnellement considéré comme plutôt masculin, et incompatible avec un rôle féminin. Comme l'ont montré les travaux sur la construction des rôles sexués, cette socialisation différenciée affecte les attitudes et les comportements de sujets. Dans cette étude menée auprès de 782 adolescents (361 garçons et 461 filles) âgés en moyenne de 15,4 ans (ET = 0,83 ans), il est supposé que leurs choix et leurs rejets concernant les activités physiques et sportives dans le cadre des cours d'E.P.S. sont médiés par leurs scores aux sous-échelles «masculine » et «féminine » de la version française de l'Inventaire des Rôles Sexués de Bem (Fontayne, Sarrazin, et Famose, 2000). Les résultats sont discutés à la lumière des divers cadres théoriques définissant les relations entre les différents " rôles de genre » et, en particulier, du modèle " additif et différentiel » de l'androgynie (Marsh et Byrne, 1991).

Mots clés : Masculinité, Féminité, Choix, Activités Sportives, Modèle.

\section{ABSTRACT}

The domain of sports and physical activities is traditionally considered as rather male, and incompatible with a feminine role. As showed in the studies on the construction of sexual roles, this differentiated socialization affects the individual's attitudes and behaviors. This study was conducted on 782 teenagers ( 361 males, 421 females). Mean age for the participants was 15.4 years old $(\mathrm{SD}=.83)$. It was further predicted that physical activity choices in Physical Education depend upon individuals' score on both masculinity and feminity subscales of the French short version of the Bem Sex Role Inventory (Fontayne, Sarrazin, \& Famose, 2000). Results are then dicussed according to different theoretical approaches of the relationships between gender roles. The differentiated additive androgyny model (Marsh \& Byrne, 1991) is more particularly examined.

\footnotetext{
* Centre de Recherches en Sciences du Sport, Université de Paris XI-Orsay, France. Division STAPS, Bat 335 91405 Orsay cedex France - tel : 33(0)1 69156276 - email : paul.fontayne@wanadoo.fr

** Équipe de Recherches sur l'Offre Sportive, Université de Grenoble I, France.
} 
Key words : Masculinity, Feminity, Choice, Sport activities, Model.

\section{Introduction}

Depuis plus d'une vingtaine d'années, l'idée que chaque culture oriente et encourage certaines conduites, traits et activités considérés comme des caractéristiques propres à chacun des sexes est l'hypothèse fondamentale qui soustend la conceptualisation et la mesure des rôles sociaux sexuellement typés. C'est la raison pour laquelle, les concepts de masculinité $(\mathrm{M})$ et de féminité $(\mathrm{F})$ sont utilisés pour faire référence aux rôles ou aux traits psychologiques attachés respectivement aux hommes et aux femmes. Ces différents rôles, une fois intériorisés, constituent des schémas de «soi » liés au genre (Bem, 1981, Cross et Madson, 1997) qui servent de filtres cognitifs pour interpréter les événements et orienter les conduites.

Les différents modèles théoriques définissant les relations entre les différents « rôles de genre $»^{1}$ sont initialement au nombre de quatre (Hall et Taylor, 1985 ; Marsh et Byrne, 1991 ; Spence et Hall, 1996 ; Taylor et Hall, 1982 ; Withley, 1983, 1984). À la base de chacun de ces modèles, nous trouvons une interprétation des résultats d'analyses de variance (ANOVAs) ou d'analyses de régression qui essayent d'identifier l'influence respective de la masculinité et de la féminité sur une ou plusieurs variables. Nous allons brièvement les résumer.

1. Le modèle traditionnel (modèle de la congruence²) de la représentation des rôles sexués est basé sur l'hypothèse que la masculinité et la féminité représentent chacun l'un des deux pôles opposés d'un même continuum. Il s'agit d'une conception bipolaire dans laquelle masculinité et féminité s'excluent mutuellement. Selon cette représentation, l'acquisition des rôles féminins et masculins doit être «congruente » avec le sexe biologique : masculin pour les hommes, féminin pour les femmes. Dans une telle acception, cela suppose que les effets principaux de $\mathrm{M}$ et de $\mathrm{F}$ interagissent avec le sexe biologique dans la même direction.

Cependant, il existe maintenant un large consensus autour de l'hypothèse de Constantinople (1973) selon laquelle masculinité et féminité constituent deux dimensions indépendantes (conception bidimensionnelle). Chaque individu peut posséder un niveau plus ou moins élevé de ces deux traits, quel que soit son sexe biologique (pour une revue consulter Alain, 1996 ; Blanchard-Fields, Suhrer-Roussel, et Hertzog, 1994 ; Marsh et Myers, 1986). C'est le modèle de l'androgynie psychologique. Il existe plusieurs variantes de ce dernier.

1 Gender role.

2 On trouve également ce modèle sous le nom de « sex-typed model «. 
2. La première approche est appelée « modèle de la masculinité ». Celui-ci est basé sur une série d'observations empiriques indiquant qu'à chaque fois qu'une relation positive est trouvée entre l'androgynie et une variable, celle-ci peut être attribuée à sa composante « masculinité » tandis que l'effet du facteur " féminité » est soit moins important, soit négligeable. Marsh et Byrne (1991) rapportent que toutes les revues de littérature et méta-analyses vont dans le sens de cette analyse. Hurtig et Pichevin (1985) ainsi que Lorenzi-Cioldi (1988, 1994) soulignent également que, contrairement à la conceptualisation de Bem (1974), le «masculin » et le "féminin » ne sont pas, dans nos sociétés, placés au même niveau de la hiérarchie sociale. La « domination masculine » (Bourdieu, 1998) sur le féminin est telle que seule la possession des attributs liés à la définition de la masculinité semble importante pour justifier des différences constatées entre les sujets quant à leurs comportements, leurs statuts sociaux, ou bien même leur orientation scolaire ou professionnelle. Ce modèle implique donc qu'il existe un effet principal significatif de $M$, mais pas d'effet principal de $\mathrm{F}$.

3. Le second modèle est le « modèle additif de l'androgynie ». Dans celui-ci la masculinité et la féminité contribuent indépendamment à la prédiction du comportement du sujet, et l'androgynie représente l'addition des effets liés à ces deux concepts. Dans cette approche, l'effet de l'androgynie psychologique est avéré quand, par exemple, dans une analyse de variance, les effets principaux de $\mathrm{M}$ et de $\mathrm{F}$ sont significatifs (on appelle également cette approche le « modèle des effets principaux $»^{3}$ ).

4. Enfin, le dernier modèle de l'androgynie psychologique est le «modèle interactif $»^{4}$. Dans celui-ci, l'effet androgyne est conceptualisé comme l'interaction des effets produits par les deux dimensions indépendantes que sont la masculinité et la féminité. Il faut, dans le cas d'une analyse de variance, que l'effet de l'interaction entre $M$ et $F$ soit significatif, ou bien que dans une analyse de régression multiple, le produit $\mathrm{MxF}$ ait une contribution supérieure pour la prédiction de la variable étudiée à la contribution de M ou de F pris séparément. Hall et Taylor (1985) distinguent deux variantes à cette conceptualisation de l'androgynie psychologique, un "modèle interactif équilibré $»^{5}$ dans lequel seule l'interaction MxF doit être significative (i.e., sans effet principal de $\mathrm{M}$ ou de F) et un " modèle interactif émergeant ${ }^{6}$ dans lequel il est présumé des effets significatifs de $\mathrm{M}, \mathrm{F}$ et $\mathrm{MxF}$.

Trois principales critiques peuvent être faites aux études menées avec ces modèles. Premièrement, la plupart des études ne tiennent pas compte de

\footnotetext{
Main effect model.

Interactive model.

Balance interactive model.

Emergent interactive model.
} 
la position asymétrique du masculin et du féminin dans nos sociétés (Lorenzi-Cioldi, 1988, 1994), et qu'il est souvent plus « désirable » pour un sujet de montrer qu'il possède des qualités masculines. Ainsi, le biais affecté à des réponses empreintes de désirabilité sociale peut non seulement affecter la corrélation entre $\mathrm{M}$ et $\mathrm{F}$ mais surtout affecter les relations qu'entretiennent ces deux construits avec les variables mesurées ou manipulées par le ou les chercheurs. En deuxième lieu, la plupart des études conduites sont basées sur un plan expérimental 2 X 2 (Hall et Taylor, 1985 ; Whitley, 1983) dans lequel M et F sont dichotomisés en deux catégories « haut » et «bas ». Cette approche est critiquable en soi car elle introduit plus de variance systématique dans les réponses des sujets aux sous-échelles M et F. Spence (1984) souligne déjà les effets non linéaires de $\mathrm{M}$ et de $\mathrm{F}$ ainsi que de l'interaction $\mathrm{MxF}$, et conclut à l'inadéquation de plan $2 \times 2$. L'utilisation d'une catégorisation dichotomique des scores $\mathrm{M}$ et $\mathrm{F}$ ne semble pas souhaitable et d'autres approches plus sophistiquées devront être utilisées.

Enfin, les diverses études menées, aussi bien sur les effets de l'identité de genre, que celles sur les structures factorielles des instruments de mesure de la masculinité et de la féminité (pour revue voir, Blanchard-Fields et coll., 1994 ; Spence et Hall, 1996) sont empreintes de multiples biais (i.e., groupes nonéquilibrés au niveau de la variable sexe, objet d'étude androcentré, approche unidimensionnelle, inadéquation des analyses statistiques,...).

Afin de dépasser les critiques formulées à l'égard des approches précédentes, une conceptualisation plus récente de l'androgynie psychologique fut proposée : le «modèle additif et différentiel de l'androgynie $»^{7}$ (Marsh, 1987 ; Marsh et Byrne, 1991). Pour ces auteurs, si les effets des facteurs « masculinité » et « féminité » sont attendus, leur contribution relative dépend du domaine étudié, les scores aux sous-échelles $\mathrm{M}$ et $\mathrm{F}$ devant être positivement corrélés avec les domaines spécifiques avec lesquels ils sont les plus logiquement et théoriquement associés. C'est une approche de type situationnelle.

«En premier lieu, il est important de démontrer que ces patrons de relations se généralisent à travers différentes études, différents instruments et différentes populations. Deuxièmement, il est important d'opérationnaliser la manière la plus logique dont les domaines étudiés sont reliés à $\mathrm{M}$ ou à $\mathrm{F}$ » (Marsh et Byrne, 1991, p. 814).

Cependant, depuis ces propositions formulées par Marsh et Byrne (1991), peu de recherches empiriques ont été menées pour mettre à l'épreuve la validité de ce modèle

Nous nous proposons donc de conduire un travail de ce type à partir de l'étude des choix et des rejets d'activités physiques et sportives (A.P.S.) dans le cadre scolaire par des adolescents de classe de $3^{\circ}$ en collège. En effet, si dans

7 Differentiated additive androgyny model. 
notre société, de nombreuses pratiques sociales sont sexuellement typées (Maccoby, 1990), le sport ne fait pas exception à ce marquage sexuel. En général, il est considéré par les auteurs comme un domaine plutôt masculin (Davisse et Louveau, 1998 ; Harry, 1995 ; Matteo, 1986, 1988 ; Messner, 1988, 1990 ; Ryckman et Hamel, 1995). Certains auteurs ont vu là une des explications aux différences de participation et d'investissement entre les garçons et les filles (Eccles et Harold, 1991 ; Deeter, 1989, 1990). Pour ces chercheurs, ces différences qui paraissent émerger très tôt (Tap, 1985 ; Eccles, Jacobs, et Harold, 1990 ; Wigfield, Harold, Freedman-Doan, Eccles, Yoon, Arbreton, et Blumenfeld, 1997), semblent être plutôt la conséquence d'une socialisation des rôles sexués que d'une différence d'aptitudes naturelles (Coupey, 1995). L'environnement social et culturel inculquerait très tôt ce qu'il est approprié de faire en fonction du sexe. Cette socialisation aboutirait à ce que les sujets s'engagent dans des activités singulières en fonction de leur conformité aux stéréotypes de leur genre. Sur la base de l'appropriation sexuée des pratiques, de récents travaux ont corroboré l'existence d'activités sportives « masculines », " féminines » ou « appropriées aux deux sexes » (Fontayne, 1999 ; Fontayne, Sarrazin, et Famose, sous presse). Guillet, Sarrazin, et Fontayne (2000) ont également montré, en accord avec les travaux de Bem (1981), que les individus qui possèdent une forte identité de genre (i.e., Typés Masculins ou Typés Féminins) marquent une préférence pour les activités sportives qui sont congruentes avec cette identité, et que les individus dont le schème de catégorisation serait absent (i.e., Non-différenciés) sont indifférents à ces pratiques, ou que ceux qui n'utilisent pas cette dichotomie du masculin et du féminin (i.e., Androgynes) pratiquent plus aisément des activités nonconformes aux stéréotypes de leur sexe.

Confirmant globalement les résultats de quelques études existant sur l'effet du genre sur le choix des activités physiques et sportives dans le monde associatif (Colley, Roberts, et Chipps, 1985 ; Colley, Nash, O'Donnel, et Restorick, 1987 ; Engel, 1994 ; Matteo, 1986, 1988), les travaux de Fontayne (1999), de Fontayne et coll. (sous presse), et de Guillet et coll. (2000) soulèvent néanmoins un certain nombre de questions. En effet, si le fait de s'adonner à une activité sportive semble généralement lié à la "masculinité », il apparaît également que l'influence de la « féminité » n'est pas absente quand il s'agit de pratiquer des disciplines sportives perçues comme «féminines ». Il semble donc que l'hypothèse de Marsh et Byrne (1991) concernant l'influence différentielle des deux dimensions que sont la «masculinité » et la « féminité » soit fonction de l'objet étudié. De plus, beaucoup d'études menées dans le cadre du sport, ne prennent pas en compte l'ensemble des sujets d'une population (i.e., filles et garçons ; sportifs et non-sportifs). C'est pourquoi il nous semble particulièrement intéressant d'étudier les choix et les rejets d'une population $\mathrm{d}$ 'adolescents dans le cadre obligatoire par la pratique scolaire afin de pallier aux biais de certaines études antérieures.

Mais avant de formuler nos hypothèses, il nous semble nécessaire d'apporter quelques précisions sur les termes que nous avons employés jusqu'ici. 
En effet, quelques confusions peuvent naître de l'utilisation des termes de " masculinité » et de "féminité ». Comme le rappellent Spence et Hall (1996), il ne saurait être question de définir ces deux concepts à partir d'une simple mesure réalisée à partir d'un questionnaire. De nature multidimensionnelle, ces derniers englobent plus que la mesure de traits de personnalité. Même si l'instrument utilisé ici (i.e., IRSB) est lui-même multidimensionnel (voir Blanchard-Fieds et coll., 1994 ; Fontayne, Sarrazin, et Famose, 2000 ; Marsh et Myers, 1986) les notions de "masculinité » et de «féminité » ne sont utilisées dans cette recherche que pour labéliser deux ensembles de traits psychologiques perçus comme socialement désirables dans notre société pour caractériser le féminin et le masculin.

Dans le cadre que nous venons de définir, nous poserons donc un certain nombre d'hypothèses afin d'éprouver la validité écologique du modèle « additif et différentiel » de l'androgynie psychologique (Marsh, 1987 ; Marsh et Byrne, 1991). Si celle-ci est avérée, les choix et les rejets des A.P.S. dans le cadre obligatoire de l'Éducation Physique et Sportive (E.P.S.) à l'école devrait être liés de manière différentielle à la «masculinité » et à la «féminité » suivant que les activités sportives sont perçues comme "masculines», "féminines », ou « appropriées aux deux sexes ». Conformément à ce que proposent Marsh et Byrne (1991), nous devrions constater que :

Hypothèse 1 : La relative contribution de $\mathrm{M}$ et de $\mathrm{F}$ aux choix et aux rejets des activités physiques et sportives varie de manière substantielle en fonction $\mathrm{du}$ « typage sexuel » des différentes A.P.S. proposées aux élèves.

Hypothèse 2 : Les contributions de $\mathrm{M}$ et de $\mathrm{F}$ sont marquées dans le sens le plus « logiquement et le plus théoriquement » attendu concernant les choix des A.P.S. « masculines » et les rejets des A.P.S. « féminines ».

Hypothèse 3 : Les contributions respectives de $\mathrm{M}$ et de $\mathrm{F}$ sont plus équilibrées en ce qui concerne les choix et les rejets des A.P.S. «appropriées aux deux sexes ».

\section{Méthode}

\section{Participants}

La population était constituée de 782 élèves de classes de $3^{\circ}$ de sept collèges de la banlieue ouest de Paris et d'un collège de la Drôme. Ces élèves étaient âgés en moyenne de 15,4 ans $(\mathrm{ET}=0,83)$. Ces 361 garçons ( 254 pratiquaient un sport dans une association en dehors de l'école) et ces 421 filles (146 pratiquaient un sport dans une association en dehors de l'école) ont tous été volontaires pour remplir les questionnaires dont la présentation avait auparavant été soumise à autorisation parentale. 


\section{Procédure}

Les questionnaires étaient administrés dans une classe, à la fin d'un cours sous la responsabilité conjointe du professeur d'Éducation Physique et Sportive de la classe et d'un chercheur en psychologie du sport formé à la passation des questionnaires. La durée approximative pour répondre aux questionnaires était d'environ 20 minutes.

\section{Mesures}

Masculinité, féminité. L'évaluation de ces deux dimensions a été faite par le score aux sous-échelles mesurant la «masculinité » et la « féminité » de la version courte pour adolescents français de l'IRSB (Fontayne et coll., 2000). Le questionnaire est constitué de 18 items (8 pour la sous-échelle masculine et 10 pour la sous-échelle féminine), représentant chacun un attribut de personnalité. Certains sont caractéristiques de traits féminins [e.g., Je suis toujours prêt(e) à écouter les autres ; je suis affectueux (se)] et d'autres des traits masculins [e.g., Je me comporte en chef ; Je suis sûr(e) de moi]. Le sujet est invité à indiquer pour chacun d'eux, s'il est particulièrement approprié pour le décrire. Il utilise pour cela une échelle de type Likert en 7 points : (1) «Jamais vrai » et (7) « Toujours vrai». Dans les travaux antérieurs menés avec plus de 1200 adolescents (Fontayne et coll., 2000), cette version raccourcie de l'IRSB a témoigné d'une bonne validité de construit, et les deux sous-échelles ont démontré une consistance interne élevée et une fiabilité test-retest acceptable. Des arguments en faveur de la validité concomitante, et de la validité prédictive du questionnaire ont également été rapportés. Pour cet échantillon de sujets, les coefficients alpha (Cronbach, 1951) sont de .81 et de .86 respectivement pour les sous-échelles masculine et féminine.

Perception sexuée des activités physiques et sportives. Afin d'évaluer la perception sexuée des A.P.S., nous avons présenté, par ordre alphabétique, une liste de 15 pratiques sportives. Choisies sur la base des programmations d'E.P.S. des différents collèges et de travaux antérieurs (pour revue, voir Fontayne, 1999) les activités ont été classées comme suit :

- activités masculines : Basket-ball, Boxe, Football, Rugby, Saut à la perche

- activités féminines : Danse, Équitation, Gymnastique Rythmique, Gymnastique, Natation synchronisée

- activités appropriées au deux sexes : Badminton, Course d'orientation, Natation, Tennis, Volley-ball.

Il était demandé au sujet d'indiquer comment il percevait ces activités sportives sur une échelle à 7 points : (1) Très masculine, (4) À la fois masculine et féminine, (7) Très féminine. Cette procédure a été utilisée par de nombreux auteurs analysant la perception sexuée des activités physiques 
ou des disciplines scolaires (Archer et Freedman, 1989 ; Archer et Macrae, 1991 ; Colley et coll., 1987 ; Ignico, 1989 ; Koivula, 1995 ; Matteo, 1986 ; Mead et Ignico, 1992 ; Pellett et Harrison, 1992 ; Pellett et Ignico, 1993 ; Salminen, 1990). Les sports dont les scores moyens sont entre 3.5 et 4.5 sont considérés comme "appropriés aux deux sexes », ceux dont les scores moyens sont compris entre 1 et 3.5 , et entre 4.5 et 7 sont respectivement considérés comme «masculins » et « féminins » (Ignico, 1989 ; Koivula, 1995 ; Matteo, 1986 ; Mead et Ignico, 1992 ; Pellett et Harrison, 1992 ; Pellett et Ignico, 1993).

TABLEAU I.

Moyennes et écart-types des scores à l'échelle de perception sexuée des activités physiques et sportives pour la population entière, pour le groupe des garçons $(\mathrm{N}=361)$, et pour le groupe des filles $(\mathrm{N}=421)$.

\begin{tabular}{|c|c|c|c|c|c|c|}
\hline Pop. Totale & \multicolumn{2}{|c|}{ Garçons } & \multicolumn{2}{|c|}{ Filles } & \multirow[b]{2}{*}{ M } & \multirow[b]{2}{*}{ ET } \\
\hline Activités sportives & M & ET & M & ET & & \\
\hline \multicolumn{7}{|l|}{ Activités masculines } \\
\hline Basket-ball & 2.87 & 1.20 & 2.58 & 1.23 & 3.12 & 1.12 \\
\hline Boxe & 1.55 & 1.08 & 1.45 & 1.02 & 1.65 & 1.12 \\
\hline Football & 2.10 & 1.25 & 1.94 & 1.25 & 2.24 & 1.23 \\
\hline Rugby & 1.58 & 1.00 & 1.44 & 0.93 & 1.70 & 1.05 \\
\hline Saut à la perche & 2.80 & 1.26 & 2.76 & 1.30 & 2.83 & 1.22 \\
\hline \multicolumn{7}{|l|}{ Activités féminines } \\
\hline Danse & 6.33 & 1.07 & 6.39 & 1.08 & 6.26 & 1.05 \\
\hline Équitation & 4.68 & 1.05 & 4.78 & 1.18 & 4.62 & 0.90 \\
\hline Gymnastique Rythmique & 6.46 & 1.08 & 6.43 & 1.18 & 6.48 & 0.99 \\
\hline Gymnastique & 5.34 & 1.34 & 5.21 & 1.40 & 5.44 & 1.28 \\
\hline Natation Synchronisée & 6.00 & 1.21 & 5.92 & 1.27 & 6.06 & 1.14 \\
\hline \multicolumn{7}{|c|}{ Activités appropriées aux deux sexes } \\
\hline Badminton & 4.06 & 0.92 & 3.92 & 0.93 & 4.17 & 0.89 \\
\hline Course d'orientation & 3.96 & 0.76 & 3.96 & 0.78 & 3.95 & 0.73 \\
\hline Natation & 4.09 & 0.80 & 4.07 & 0.96 & 4.10 & 0.63 \\
\hline Tennis & 3.96 & 0.60 & 3.88 & 0.69 & 4.02 & 0.50 \\
\hline Volley-ball & 4.11 & 0.83 & 4.03 & 0.85 & 4.18 & 0.80 \\
\hline
\end{tabular}

Afin d'éprouver la validité du classement de ces différents sports dans les catégories précédemment évoquées, nous avons vérifié si les scores attribués par les sujets étaient conformes à nos attentes. Il fallait, pour que la catégorisation soit considérée comme valide, que les activités sportives soient ordonnées de manière similaire à l'hypothèse de classement, 
et ceci, par le groupe de sujets dans sa totalité, par les garçons, et par les filles. Les résultats, reportés dans le tableau I, montrent que cette classification a priori des activités physiques et sportives est bien analogue à la perception des sujets de notre population d'étude.

La perception de la difficulté. La liste alphabétique des 15 A.P.S. (5 masculines, 5 féminines, 5 appropriées aux deux sexes) fut présentée aux sujets. Ils devaient indiquer quel était pour eux le degré de difficulté de chaque sport sur une échelle en 7 points : (1) Très facile pour moi, (4) De difficulté moyenne, (7) Très difficile pour moi.

La validité de ce type de mesure de la difficulté perçue et de sa liaison avec la perception masculine - appropriée aux deux sexes - féminine d'une activité est reportée dans plusieurs articles (Archer, 1989 ; Archer et Freedman, 1989 ; Archer et Macrae, 1991 ; Granleese, Trew, et Turner, 1988). L'intérêt de ces mesures pour la compréhension de la motivation à la pratique de certaines A.P.S. dans le cadre scolaire a été démontré par Famose, Sarrazin et Cury (1995). Pour chaque sujet, une moyenne des scores de difficulté attribués à chaque groupe de sports (i.e., masculins, féminins, appropriés aux deux sexes) a été calculée. Les moyennes pour chaque groupe d'activités sportives ont été utilisées dans les analyses statistiques. Les coefficients alpha (Cronbach, 1951) sont de .72 pour les activités masculines, de .76 pour les activités féminines, et de $.64^{8}$ pour les activités appropriées aux deux sexes.

Intérêt de l'activité. La même procédure (Archer et Macrae, 1991) fut reproduite concernant la mesure de l'intérêt porté à la pratique des différentes activités physiques et sportives déjà proposées. Les sujets devaient reporter leurs réponses sur une échelle en 7 points (1) Pas du tout intéressant, (4) Modérément intéressant, (7) Vraiment très intéressant.

Pour chaque sujet, une moyenne des scores d'intérêt attribués à chaque groupe de sports (i.e., masculins, féminins, appropriés aux deux sexes) a été calculée. Les moyennes pour chaque groupe $\mathrm{d}^{\prime}$ activités sportives ont été utilisées dans les analyses statistiques. Les coefficients alpha (Cronbach, 1951) sont de .71 pour les activités masculines, de .81 pour les activités féminines, et de $.64^{9}$ pour les activités appropriées aux de sexes.

Le choix des activités physiques et sportives. Il a été demandé aux sujets d'indiquer, parmi les 15 A.P.S. déjà présentées dans les autres questionnaires,

8 Le coefficient alpha de cette échelle peut être considéré comme problématique par quelques chercheurs. Cependant, comme noté par Cronbach (1951), étant donné un petit nombre d'items, des alphas bas peuvent sous-estimer les intercorrélations entre les items qui sont la base de la consistance interne. Étant donné ces mêmes intercorrélations cette échelle produirait un alpha de .70 s'il y avait 6 A.P.S.. Avec des échelles de cet type, l'adéquation du modèle de mesure sousjacent est généralement plus indicative de la qualité de construit de la mesure que la consistance interne (Pedhazur et Schmelkin, 1991 ; voir aussi Vallerand, Fortier, et Gay, 1997 pour une argumentation similaire). Les résultats de modèles d'équations structurelles reliant les mesures réalisées sur ces 5 A.P.S. à un facteur latent ont indiqué une bonne validité de celles-ci.

9 Voir note précédente. 
quelles étaient les 5 qu'ils préféreraient, ainsi que les cinq qu'ils aimeraient le moins pratiquer dans le cadre de leurs cours d'E.P.S.. Il fut relevé, pour chaque sujet, le nombre (pouvant aller de 0 à 5) des choix et des rejets pour chaque groupe d'activités (i.e., féminines, masculines, appropriées aux deux sexes). Ces données seront utilisées dans les analyses statistiques.

\section{Traitements statistiques}

Afin d'éprouver nos hypothèses, nous avons eu recours à une analyse des pistes causales ${ }^{10}$, à l'aide du logiciel LISREL 8 (Jöreskog et Sörbom, 1993) et à partir d'une matrice de covariance asymptotique. La méthode d'estimation est celle des moindres carrés pondérés ${ }^{11}$. En effet, certaines échelles de mesures ne pouvant être assimilées à des variables strictement continues (i.e., nombre des choix et des rejets), le logiciel PRELIS (Jöreskog et Sörbom, 1993) génère des corrélations polychoriques et une matrice de covariance asymptotique lui correspondant. Les coefficients de pistes obtenus sont des coefficients de régression partielle réduits, dont la valeur quantifie le poids d'une variable sur une autre, quand l'influence des variables situées en amont dans le modèle est maintenue constante (pour davantage d'explications sur les analyses des pistes causales et les modèles structuraux, voir Bacher, 1987, 1988 ; Jöreskog et Sörbom, 1993). Pour résumer, ce type d'analyse permet de distinguer le type d'effet (positif ou négatif) existant entre les variables, d'estimer leur importance relative, et de déterminer par quelle voie (directe ou indirecte) chaque variable influence la suivante. L'utilisation d'une telle procédure semble satisfaire aux exigences d'approche statistique plus sophistiquée évitant la dichotomisation des scores $\mathrm{M}$ et $\mathrm{F}$ formulées par plusieurs auteurs et rappelées dans notre introduction.

\section{Résultats}

\section{Le choix des activités physiques et sportives}

Le principal objectif poursuivi dans cette étude est d'évaluer l'influence respective de la «masculinité » et « féminité » sur l'intérêt perçu, la difficulté perçue, ainsi que les choix et les rejets de certaines A.P.S.. Une relation entre chacune des différentes variables mesurées est donc proposée dans le modèle général de pistes causales éprouvé. Le poids et la significativité de chacune des relations seront discutés plus tard à la lumière des différents modèles théoriques évoqués en introduction.

\footnotetext{
10 Path analysis.

11 Weight least squares.
} 
Les principaux résultats ${ }^{12}$ concernant les choix des sujets sont reportés dans la figure 1.

Les activités "masculines ». La corrélation entre les sous-échelles $\mathrm{M}$ et $\mathrm{F}$ de la version courte de l'IRSB (Fontayne et coll., 2000) est quasiment nulle. Les deux dimensions peuvent donc être considérées comme indépendantes.

Les coefficients de pistes causales (voir figure 1.a) nous indiquent que les deux dimensions $\mathrm{M}$ et $\mathrm{F}$ ne sont pas directement reliées à la valeur d'intérêt que les sujets accordent à ce type d'activités sportives. Par contre la difficulté perçue de cette classe d'activité est reliée de manière positive à la sous-échelle F (i.e., plus le sujet pointe haut sur l'échelle de "féminité », plus il perçoit les activités masculines comme difficiles), et de manière négative à la sous-échelle $M$ (i.e., plus le sujet à un score de « masculinité » élevé, moins il perçoit les activités masculines comme difficiles). La difficulté perçue semble médier de manière forte et négative l'intérêt perçu (i.e., plus les activités sont perçues comme difficiles, moins les sujets semblent éprouver de l'intérêt pour ces activités). Le nombre des choix est relié de manière négative à la difficulté perçue et à la « féminité » (i.e., plus la difficulté perçue est importante ou plus le score à la sous-échelle "féminité » est élevé, moins les sujets choisissent d'activités masculines), de manière positive à la valeur d'intérêt. Il n'y a pas de liaison directe entre la «masculinité » et le nombre des choix. La part de variance expliquée concernant le choix des activités «masculines » est de $50 \%$.

Les activités "féminines ». La sous-échelle $\mathrm{M}$ est ici reliée (voir figure 1.b) de manière négative au choix d'activités sportives féminines. Les liaisons avec la difficulté perçue et l'intérêt perçu ne sont pas significatives. Quant à la sous-échelle F, elle influence de manière positive l'intérêt perçu et le nombre des choix, tandis que la liaison entre cette sous-échelle et la difficulté perçue est négative. Le choix des activités « féminines » est influencé de manière positive par la valeur d'intérêt, et de manière négative par la difficulté perçue. L'intérêt perçu est encore lié négativement à la difficulté perçue. La part de variance expliquée par l'ensemble des variables concernant le choix des activités « féminines » est de $43 \%$.

Les activités "appropriées aux deux sexes ». La sous-échelle F influence de manière positive l'intérêt perçu pour ces activités, ainsi que le nombre des choix (voir figure 1.c). Le coefficient de piste causale avec la difficulté perçue n'est pas significatif. La sous-échelle M n'a pas de liaison significative avec la valeur d'intérêt, tandis qu'elle est reliée négativement à la difficulté perçue et positivement au nombre des choix. La liaison entre la

12 Il s'agira bien de discuter des différents modèles de l'androgynie psychologique et non pas de la validité du modèle présenté ici, celui-ci n'étant envisagé que pour illustrer notre réflexion. D'ailleurs, le modèle étant « saturé «, il présente des indices d'adéquation parfaits dont l'analyse et la discussion n'auraient aucun sens (pour plus de détails, voir. Bacher, 1987, 1988 ; Jöreskog et Sörbom, 1993). 
difficulté et l'intérêt perçu est négative. L'intérêt perçu exerce une influence positive sur le nombre de choix. La liaison entre la difficulté perçue et le choix des activités n'est pas significative. La part de variance expliquée concernant le choix des activités « appropriées aux deux sexes » est de $19 \%$.
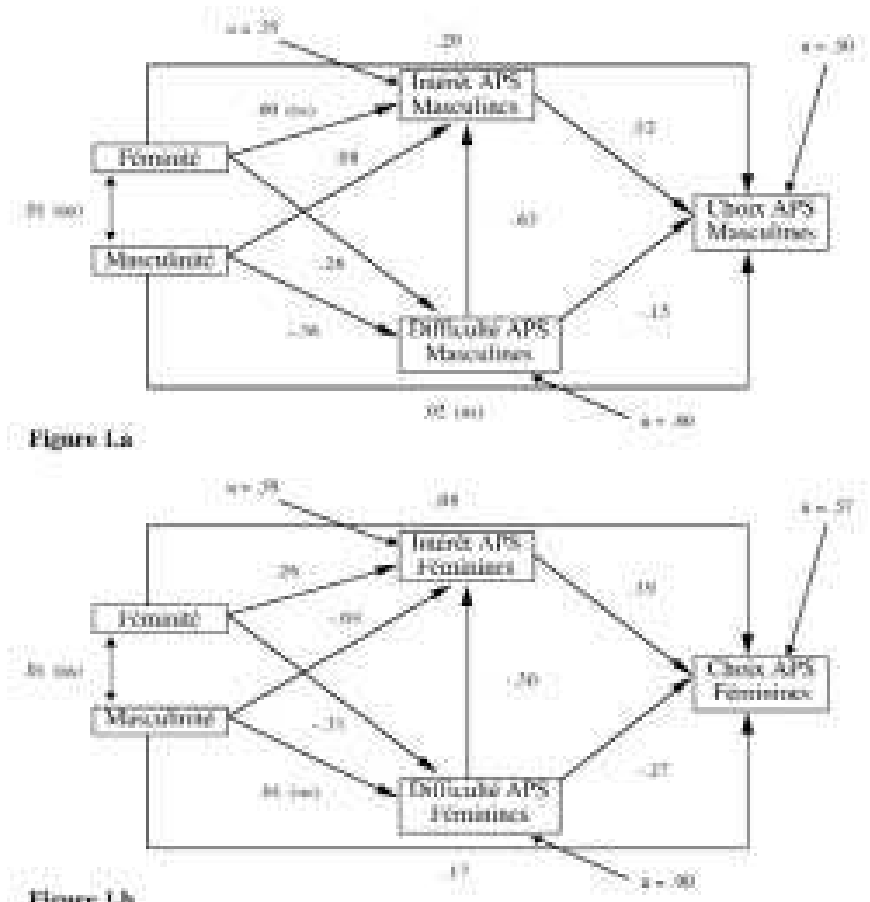

Higare is

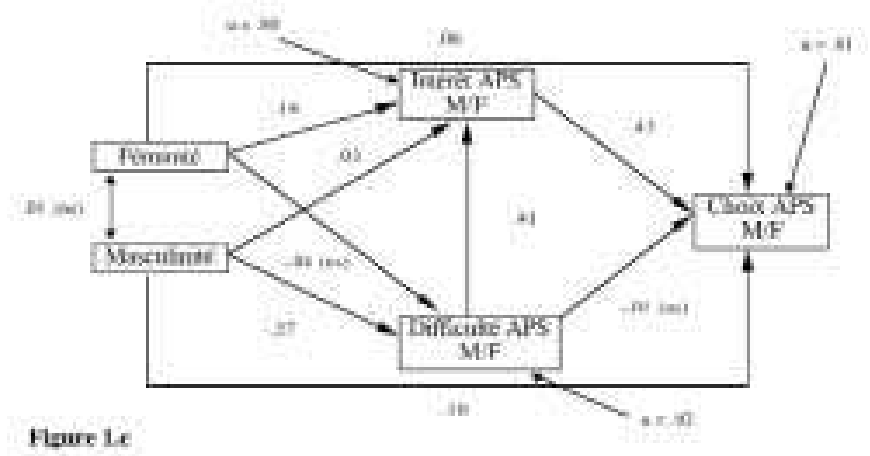

FIGURE 1

Modèles d'analyse des pistes causales du choix des activités physiques et sportives en Éducation Physique et Sportive (coefficients standardisés). 


\section{Le rejet desactivités physiqueset sportives}

Les activités «masculines ». Les sous-échelles F et M n'ont pas d'influence directe sur l'intérêt perçu (i.e., coefficients de pistes causales non-significatifs), alors qu'elles ont une influence respectivement positive et négative sur la difficulté perçue des activités sportives (voir figure 2.a). Également, la sous-échelle $\mathrm{M}$ n'a pas de liaison directe significative avec le rejet des A.P.S., tandis que la liaison entre la sous-échelle $\mathrm{F}$ et le rejet de ces mêmes activités est positive et significative. La difficulté perçue est toujours liée de manière négative à la valeur d'intérêt. Le rejet des activités sportives «masculines » est lié de manière négative à l'intérêt perçu et de manière positive à la difficulté perçue. La part de variance expliquée pour le nombre des rejets des activités «masculines » est de $66 \%$.

Les activités "féminines ». La sous-échelle M n'a pas de liaison significative avec la difficulté ou l'intérêt perçu pour les activités sportives "féminines ", tandis qu'une liaison positive existe avec le nombre de rejet de ces A.P.S. (voir figure 2.b). La sous-échelle $\mathrm{F}$ est liée de manière positive à la valeur d'intérêt, et de manière positive à la difficulté perçue et le nombre des rejets. La difficulté perçue influence de manière négative l'intérêt perçu pour les activités sportives féminines. Le nombre des rejets est liée positivement à la difficulté perçue, et de manière négative à l'intérêt perçu. La part de variance expliquée par l'ensemble des variables concernant la fréquence le nombre de rejets des activités « féminines » est de $47 \%$.

Les activités "appropriées aux deux sexes ». Dans ce cas, la sous-échelle M n'a pas de liaison significative avec l'intérêt perçu et le nombre des rejets concernant les «activités appropriées » aux deux sexes, mais on trouve une liaison négative avec la difficulté perçue (voir figure 2.c). À l'inverse, nous constatons une liaison positive entre la sous-échelle $\mathrm{F}$ et l'intérêt perçu et le nombre des rejets, tandis que la liaison avec la difficulté perçue n'est pas significative. La difficulté perçue est liée négativement à l'intérêt perçu pour ces A.P.S.. Le nombre des rejets est influencé positivement par la difficulté perçue, et négativement par la valeur d'intérêt. La part de variance expliquée par l'ensemble des variables pour le nombre de rejets des activités « appropriées aux deux sexes » est de $16 \%$. 


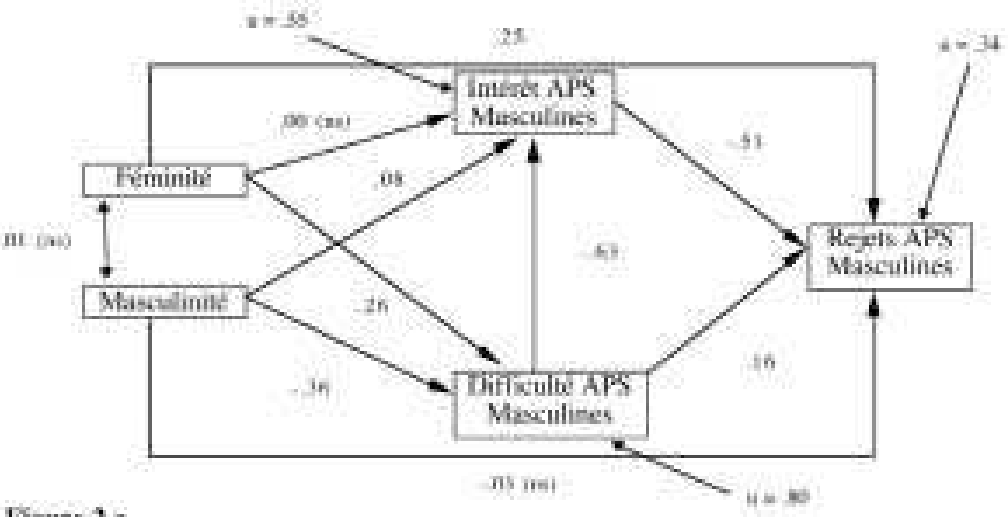

Fiqure 2a

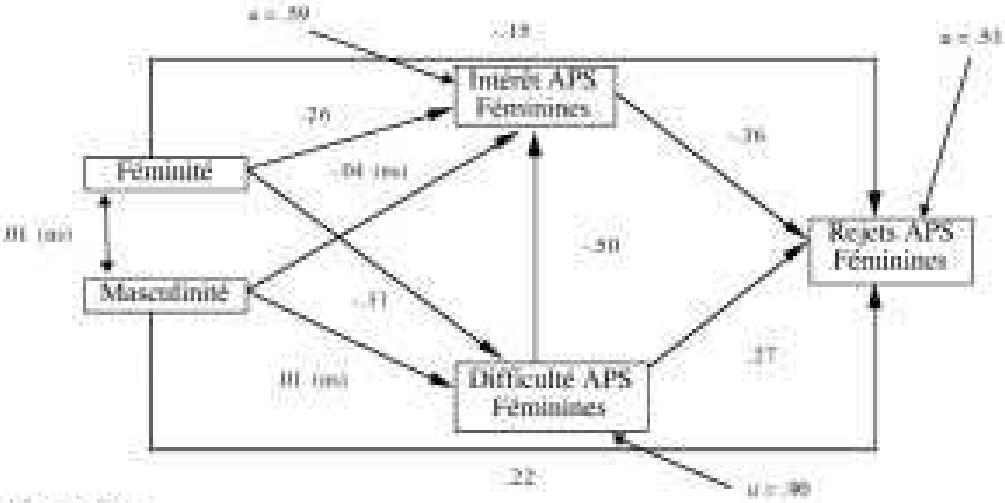

Fieure 2.h

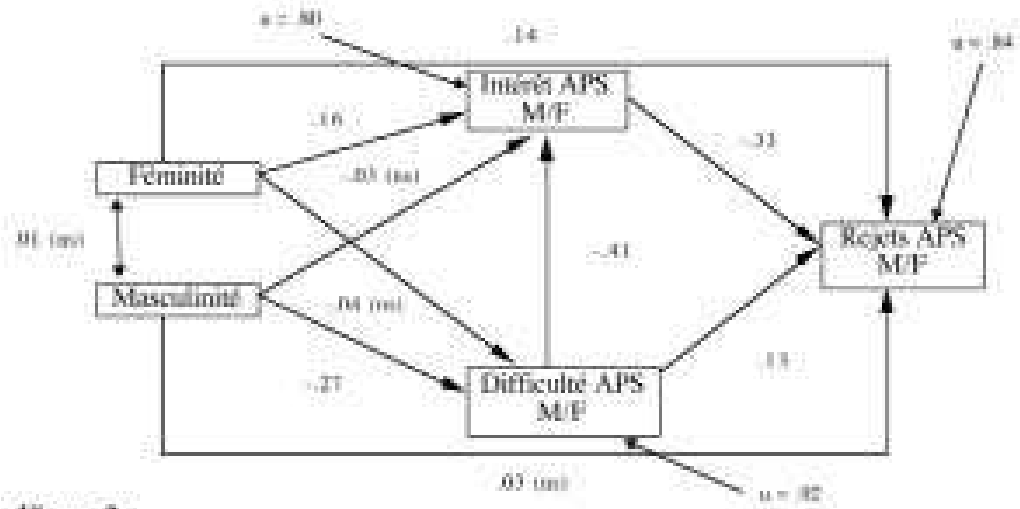

Hegure $2, e$

FIGURE 2

Modèles d'analyse des pistes causales du rejet des activités physiques et sportives en Éducation Physique et Sportive (coefficients standardisés). 


\section{Discussion}

L'objectif principal de ce travail était de tester de manière empirique la validité du modèle « additif et différentiel » de l'androgynie psychologique avancé par Marsh et Byrne (1991). Pour cela nous attendions à montrer que : (a) la relative contribution de $\mathrm{M}$ et de $\mathrm{F}$ aux choix et aux rejets des activités physiques et sportives varie de manière substantielle en fonction du «typage sexuel » des différentes A.P.S. proposées aux élèves, (b) les contributions de $\mathrm{M}$ et de $\mathrm{F}$ sont marquées dans le sens le plus "logiquement et le plus théoriquement » attendu concernant les choix des A.P.S. "masculines » et les rejets des A.P.S. "féminines ", et (c) les contributions respectives de $\mathrm{M}$ et de F sont plus équilibrées en ce qui concerne les choix et les rejets des A.P.S. " appropriées aux deux sexes ».

Nos résultats valident globalement les hypothèses présentées et nous permettent de discuter des différents modèles théoriques définissant les relations entre les différents « rôles de genre ». Ainsi, le modèle traditionnel de la « congruence» ne peut être accepté. En effet, nous constatons ici, conformément à l'hypothèse originale de Constantinople (1973), et aux résultats avancés dans la validation française de l'IRSB (Fontayne et coll., 2000), que les dimensions masculines et féminines sont indépendantes. Les corrélations mises en évidence entre les deux sous-échelles sont quasiment nulles $(\phi=.01)$ (voir figures 1 et 2). Néanmoins, même si les données de cet échantillon peuvent aller dans ce sens, nous nous garderons bien de conclure à l'orthogonalité de ces deux concepts, car les résultats sont contradictoires avec ceux d'autres études menées auprès d'adolescents français (Fontayne et coll., sous presse, 2000 ; Guillet et coll., 2000) qui montrent des corrélations, certes faibles, mais significatives entre ces deux concepts. Campbell et Gillapsy (1997) indiquaient déjà que $\mathrm{M}$ et $\mathrm{F}$ sont «essentiellement orthogonaux » (p. 420), la corrélation pouvant varier avec les échantillons même si les construits demeurent euxmêmes invariants.

De même, il ne semble pas que les données analysées supportent le modèle de la «masculinité ». En effet, si celui-ci était avéré, les scores obtenus pas les sujet à la sous-échelle masculine devraient influencer, soit directement, soit indirectement (i.e., par l'intermédiaire des variables médiatrices « difficulté perçue » et "intérêt ») les choix et les rejets des A.P.S. présentées. L'influence de la sous-échelle féminine devant être nulle ou négligeable. Ceci n'est le cas dans aucun des modèles présentés. Même si la significativité de certaines liaisons doit être examinée avec prudence (par exemple, les poids factoriels des liaisons directes entre la "féminité » et les choix des A.P.S. " féminines » $[\beta=.08]$ ou " appropriées aux deux sexes » $[\beta=-.06]$; voir figures 1.b et 1.c) car elle ne pourrait être due qu'au nombre élevé de sujets de l'étude, le poids important des liaisons (parfois directes, parfois avec les variables médiatrices) entre $\mathrm{F}$ et les choix ou les rejets de chacune des familles 
d'A.P.S. ne saurait faire qu'on tienne le rôle de la «féminité » comme nul ou négligeable. Bien que le modèle de la "masculinité » soit le plus fréquemment évoqué par les auteurs (Colley et coll., 1985 ; Colley et coll., 1987 ; Engel, 1994 ; Matteo, 1986, 1988 ; pour une revue plus complète, voir Fontayne, 1999) quand il s'agit du domaine des activités sportives, nous avancerons deux raisons principales afin d'éclairer le fait que ce modèle ne puisse expliquer l'appétence ou l'aversion pour certaines activités sportives. Dans un premier temps, la plupart des études abordent le problème de la participation sportive dans sa globalité (i.e., sportifs versus non-sportifs), ou bien à travers des disciplines sportives qui sont majoritairement pratiquées par les hommes (par exemple : les sports collectifs). La dimension masculine est alors prégnante dans ce type d'approche qui ne se soucie pas assez de la représentation sexuée des pratiques pour les sujets. Deuxièmement, cherchant dans le modèle androgyne l'explication de la pratique sportive (ou de certaines pratiques sportives) par une population jusqu'ici minoritaire, les chercheurs excluent tous les sujets masculins, et les travaux se centrent la plupart du temps sur une population uniquement composée de femmes. C'est ignorer que le masculin et le féminin sont solidaires, et sont déterminés par une structure asymétrique, à savoir que ces deux concepts n'y ont ni une place, ni une fonction équivalente (Hurtig et Pichevin, 1985 ; Lorenzi-Cioldi, 1988). C'est ainsi que dans un contexte de mixité scolaire, le choix (ou rejet) d'une activité peut se faire parce que ce choix (ou rejet) est conforme au choix majoritaire des sujets de même sexe et permet à l'élève de ne pas être perçu(e) comme trop (ou pas assez) masculin(e) ou féminin(e). L'asymétrie masculin / féminin, concomitante de la hiérarchie sociale, est un déterminant de la structure et du mode de fonctionnement de la représentation des sexes, y compris dans les pratiques sportives «nouvelles» (Davisse, 1999). Si on élimine les sujets masculins d'une étude, on peut ainsi faire apparaître une prépondérance de la «masculinité » dans les choix de pratique sportive de la population féminine étudiée. Bien que toutes les activités ne soient pas équivalentes, être sportive, c'est être plus «active», donc plus «masculine», que les non-sportives (Fontayne et coll., sous-presse).

Parmi les autres modèles évoqués dans notre introduction, le modèle « additif et différentiel » avancé par Marsh et Byrne (1991) nous semble être le plus pertinent pour expliquer les choix et les rejets des sujets. En effet, l'analyse des données nous donne à penser que les trois conditions qui permettent de soutenir la validité du modèle (Marsh et Byrne, 1991) sont globalement respectées. En premier lieu, nous pouvons constater que l'influence respective de la «masculinité » et de la «féminité » est bien fonction du type de sport (i.e., féminin, masculin, approprié aux deux sexes). Par exemple, il existe une liaison significative entre la "féminité » et l'intérêt pour les A.P.S. "féminines » $(\beta=.26)$ (voir figures 1.b et 2.b), mais pas avec l'intérêt pour les A.P.S. «masculines » $(\beta=.00)$ (voir figures 1.a et 2.a). De la même façon, la «masculinité » est reliée différentiellement à la perception de la difficulté des A.P.S suivant qu'elles sont «masculines » $(\beta=-.36)$ ou « féminines » $(\beta=.01)$ (voir figures 
1.a,b et 2.a,b). Deuxièmement, et conformément à ce que nous attendions, l'influence qu'exerce chacun de ces deux concepts s'applique de manière congruente avec le «typage sexuel » des différents groupes d'A.P.S.. Quand il s'agit d'activités «masculines », l'effet de $\mathrm{M}$ influence (de manière directe ou indirecte) positivement les choix et négativement les rejets de ces A.P.S., tandis que nous constatons la même chose concernant l'influence de F sur les choix et rejets des activités « féminines » (voir figures 1.a,b et 2.a,b). Nous pouvons également percevoir un effet qui n'était pas évoqué dans nos hypothèses : celui du renforcement de l'action de l'échelle congruente par celui de l'échelle non-congruente. Par exemple, si nous avons plus de chances qu'un sujet choisisse plus volontiers des A.P.S. "masculines » si son score est élevé sur $M$, nous en avons encore plus si le score de ce même sujet sur F est bas. Le profil inverse ( «bas » sur M et « haut » sur F) est observé en ce qui concerne le rejet de ces mêmes activités. De même, plus le sujet est « haut » sur F et «bas » sur M, plus ses choix d'activités "féminines » seront élevés, alors que s'il est « bas » sur F et « haut » sur M, il aura tendance à rejeter en plus grand nombre ces mêmes activités. Cela s'avère conforme à la théorie du "schéma de genre » avancée par Bem (1981) et aux études antérieures réalisées sur le choix des activités sportives d'adolescents français (Fontayne et coll., sous presse ; Guillet et coll., 2000) qui montrent que les individus «typés sexuellement » ont tendance, en matière de sport, à faire des choix en adéquation avec leur orientation de genre.

Nous venons de souligner plusieurs fois que l'influence des sous-échelles $\mathrm{M}$ et $\mathrm{F}$ sur les nombres des choix et des rejets s'effectue soit directement, soit par l'intermédiaire de variables motivationnelles dont l'importance a déjà été soulignée par d'autres auteurs (Archer, 1989 ; Archer et Freedman, 1989 ; Archer et Macrae, 1991 ; Famose et coll., 1995 ; Granleese et coll., 1988). Bien que ce ne soit pas l'objectif principal de cette étude, nous pensons que le rôle de ces variables médiatrices mérite d'être brièvement évoqué. La motivation à l'accomplissement étant déterminée socialement et situationnellement, les modèles les plus récents privilégient les cognitions comme médiateurs du comportement. Beaucoup d'entre eux s'inscrivent dans la tradition des modèles « expectation-valence » (voir Famose, 1997 ; Famose et coll., 1995 ; Wigfield et Eccles, 1992) dont le point commun est de présumer que le schéma de Soi constitue une variable centrale du développement et du fonctionnement de l'individu (voir Vallerand, 1994). Le schéma de Soi lié au genre (une catégorie particulière du schéma de Soi) est donc susceptible d'affecter d'autres régulateurs socio-cognitifs comme les attentes de succès et les valeurs des tâches qui constituent les deux prédicteurs les plus proximaux des comportements liés à l'accomplissement tels que le choix d'une tâche, la persistance dans l'activité, la force de l'engagement, et bien sûr en dernier lieu, les performances réalisées. Les attentes sont fonction de la compétence perçue, de la perception de la difficulté de la tâche, des attributions causales et des stéréotypes liés au genre. Généralement, les individus typés au niveau du genre (voir Bem, 1981) ont des attentes de succès plus élevées dans les activités qui 
sont congruentes avec leur genre (i.e., activités féminines pour les individus typés féminins, et activités masculines pour les individus typés masculins), et des attentes plus faibles quand ces activités correspondent au genre opposé (Wigfield et Eccles, 1992). De plus, les individus ont plus tendance à trouver de «l'intérêt » à des activités dans lesquelles ils sont performants (e.g., Eccles et coll., 1983 ; Feather, 1988). Nos résultats vont dans le sens de telles approches, néanmoins le modèle présenté demeure trop sommaire pour en permettre une généralisation. Des approches plus complexes prenant en compte d'autres variables devront donc être privilégiées par les chercheurs travaillant à partir de modèles « expectation-valence».

Enfin, concernant notre troisième hypothèse, nous attendions que les contributions respectives de $\mathrm{M}$ et de $\mathrm{F}$ soient plus équilibrées en ce qui concerne les choix et les rejets des A.P.S. « appropriées aux deux sexes ». Ceci est le cas concernant le nombre des choix pour ce type d'A.P.S.. En effet, contrairement aux activités "masculines » et «féminines » pour lesquelles l'action des sous-échelles $\mathrm{M}$ et $\mathrm{F}$ est congruente avec la sexuation des A.P.S. (i.e., il faut être « haut » sur $\mathrm{M}$ et « bas » sur F pour choisir des activités « masculines »; il faut être « haut » sur F et «bas » sur M pour choisir des activités «féminines»), nous pouvons constater que les sujets qui font le plus le choix des activités "appropriées aux deux sexes " sont ceux qui sont à la fois «haut» sur M et «haut» sur F. Bien que les coefficients de pistes causales directes entre $\mathrm{M}$ et $\mathrm{F}$ avec le choix des activités doivent être examinés avec beaucoup de prudence, les liaisons avec les variables médiatrices montrent que plus les sujets sont « hauts » sur F plus leur intérêt pour ces activités est élevé $(\beta=.16)$, et plus ils sont « hauts » sur M moins ils perçoivent ces activités comme difficiles $(\beta=-.27)$. Moins ils perçoivent ces activités comme difficiles, plus ils éprouvent de l'intérêt pour celles-ci $(\beta=-.41)$, et plus ils ont tendance à les choisir $(\beta=.43)$. La liaison entre la difficulté perçue et le nombre des choix n'est pas significatif (voir figure 1.c). C'est donc l'action « équilibrée » et conjuguée de $\mathrm{M}$ et $\mathrm{F}$ qui permettent, en partie, d'expliquer le nombre de choix d'activités « appropriées aux deux sexes ». Selon la classification des rôles de genre utilisée par Bem (1981), ce sont les sujets « androgynes » qui seraient majoritairement susceptibles d'effectuer ces choix. Cela correspond à l'organisation du champ des pratiques sportives selon le genre mis en évidence par Fontayne et coll. (sous presse) chez des adolescents français.

Concernant le nombre de rejets des activités « appropriées aux deux sexes » (voir figure 2.c), les résultats semblent plus difficiles à interpréter, en particulier pour l'échelle de «féminité ». Pour l'échelle $M$, nous voyons que l'influence de celle-ci se fait uniquement par l'intermédiaire de la difficulté perçue, le coefficient de piste négatif $(\beta=.-27)$ nous indiquant que plus un sujet est «bas » sur l'échelle masculine, plus il perçoit les activités « appropriées au deux sexes » comme difficiles, la difficulté perçue étant reliée positivement $(\beta=.13)$ au nombre de rejets (i.e., plus je perçois les activités comme difficiles, plus je formule un nombre de rejets élevé). Pour l'autre dimension, un examen des coefficients de pistes causales reliant F à (a) l'intérêt $(\beta=.16)$, et 
(b) au nombre de rejets $(\beta=.14)$, indique tout à la fois que plus les sujets ont un score élevé sur l'échelle de "féminité " plus ils font un nombre élevé de rejets, en même temps qu'ils perçoivent ces activités physiques comme plus intéressantes. Cette contradiction n'est qu'apparente puisque le coefficient de piste négatif $(\beta=.-33)$, entre l'intérêt et le nombre de rejets nous indique que plus l'intérêt pour les activités est marqué, moins les sujets font de rejets. Néanmoins, cela ne fait qu'ajouter à la difficulté d'interprétation car cela nous révèle que les sujets qui expriment le plus grand nombre de rejets sont ceux qui auraient à la fois un score élevé sur $\mathrm{F}$ (e.g., influence directe) et un faible score sur F (e.g., influence indirecte). En réalité, cela ne fait que souligner l'action différentielle de la sous-échelle " féminité » car ces résultats sont conformes à l'étude réalisée sur des adolescents français par Fontayne et coll. (sous presse) qui montrent que ce sont principalement les sujets non-différenciés (e.g., «bas » sur M et «bas » sur F) et typés féminins (e.g., «bas » sur M et « haut » sur F) qui «évitent» la pratique sportive. Ces deux orientations sont donc présentes en même temps dans le modèle proposé. Cette influence combinée et différentielle de $\mathrm{M}$ et de $\mathrm{F}$ semble donc aller dans le sens des propositions de Marsh et Byrne (1991). Néanmoins, le faible poids de certains coefficients de pistes causales et le faible pourcentage de variance expliquée par le modèle nous suggèrent que lorsqu'il s'agit d'activités « appropriées aux deux sexes ", les scores sur M et sur F n'expliquent que partiellement le nombre des rejets. Autrement dit, il faut sans doute chercher d'autres explications que celle du schéma de Soi lié au genre, pour expliquer l'aversion (et l'appétence) pour ces activités (e.g., l'habileté perçue spécifique à cette activité, la difficulté perçue, etc.). Encore une fois, la prise en compte de modèles plus complexes intégrant un plus grand nombre de variables pourrait se révéler extrêmement féconde.

En conclusion, nous dirons donc que les données présentées dans cette étude vont dans le sens de du modèle « additif et différentiel » de l'androgynie psychologique avancé par Marsh (1987) et Marsh et Byrne (1991). Cependant, il serait sans doute intéressant d'inclure les dimensions mesurant le schéma de Soi lié au genre ( $\mathrm{M}$ et $\mathrm{F}$ ) dans un modèle plus large de l'accomplissement permettant, non seulement d'expliquer l'engagement du sujet dans une activité, mais également qui permettrait, par des études longitudinales, de mettre à jour la construction de ces deux concepts. Celle-ci pourrait être envisagée comme étroitement dépendante du développement cognitif, la définition du Soi étant avant tout individuelle et intégrant de nombreuses autres dimensions. À notre sens, l'étude des différences individuelles dans les comportements et motifs d'accomplissement dans le domaine des activités physiques et sportives ne peut se faire qu'à partir de la prise en compte des contextes culturels et socio-historiques dans lesquels se sont développées les pratiques sportives, et ceci dans une perspective dynamique et interactionniste. 


\section{Bibliographie}

ALAIN M (1996) La mesure des rôles sexuels. Bulletin de Psychologie. 424 : 396-404.

ARCHER J (1989) Childhood gender role: structure and development. The Psychologist 2 : 367-370.

ARCHER J, FREEDMAN S (1989) Gender stereotypic perception of academic disciplines. British Journal of Educational Psychology 59 : 306-313.

ARCHER J, MACRAE M (1991) Gender-perceptions of school subjects among 10-11 year-olds. British Journal of Educational Psychology 61 : 99-103.

BACHER F (1987) Les modèles structuraux en psychologie. Présentation d'un modèle : Lisrel, première partie. Le Travail Humain 50 : 347-370.

BACHER F (1988) Les modèles structuraux en psychologie. Présentation d'un modèle : Lisrel, deuxième partie. Le Travail Humain 51: 272-288.

BEM SL ( 1974) The measurement of psychological androgyny. Journal of Consulting and Clinical Psychology. 42 : 155-162.

BEM SL (1981) Gender schema theory: A cognitive account of sex-typing. Psychological Review. 88 : 354-364.

BLANCHARD-FIELDS F, SUHRER-ROUSSEL L, HERTZOG C (1994) A confirmatory factor analysis of the Bem Sex Role Inventory: Old questions, new answers. Sex Roles 30(5/6) : 423-457.

BOURDIEU P (1998) La domination masculine. Paris, Éditions du Seuil.

CAMPBELL T, GILLAPSY JA (1997) The factor structure of the Bem Sex-Role Inventory (BSRI): Confirmatory Analysis of long and short forms. Educational and Psychological Measurement. 57(1): 118-124.

COLLEY A, ROBERTS N, CHIPPS A (1985) Sex-role identity, personality and participation in team and individual sports by males and females. International Journal of Sport Psychology 16(2) : 103-112.

COLLEY A, NASH J, O'DONNEL L, RESTORICK L (1987) Attitudes of the female sex-role and sex-typing of physical activities. International Journal of Sport Psychology. 8(1) : 19-29.

CONSTANTINOPLE A (1973) Masculinity-feminity: An exception to a famous dictum? Psychological Bulletin. 80 : 389-407.

COUPEY S (1995) Pratiques d'éducation physique et sportive au CP et différences de performance entre filles et garçons. Revue Française de Pédagogie. 110 : 37-50.

CRONBACH L (1951) Coefficient alpha and internal strucure of test. Psychometrika $16: 296-334$.

CROSS SE, MADSON L (1997) Models of the self: self-construals and gender. Psychological Bulletin. 122(1) : 5-37.

DAVISSE A (1999) Les filles, point aveugle des sports, nouveaux ou anciens. Revue EPS. 277 : 80-81.

DAVISSE A, LOUVEAU C (1998) Sports, école, société. La différence des sexes. Paris, L'Harmattan.

DEETER TE (1989) Development of a model of achievement behavior for physical activity. Journal of Sport and Exercise Psychology. 11 : 13-25.

DEETER TE (1990) Re-modeling expectancy and value in physical activity. Journal of Sport and Exercise Psychology. 12 : 86-91.

ECCLES JS, JACOBS JE, HAROLD RD (1990) Gender role stereotypes, expectancy effects, and parents' socialization of gender differences. Journal of Social Issues . 46(2) : 183-201.

ECCLES JS, HAROLD R (1991) Gender differences in sport involvement: Applying the Eccles and al. model. Journal of Applied Sport Psychology. 3(1) : 7-35. 
ENGEL A (1994) Sex Roles and gender stereotyping in young women's participation in sport. Special features: Doing by degrees: Feminist undergraduate dissertations. Feminism and Psychology. $4: 439-448$.

FAMOSE J-P (1997) Motivation et performance sportive In Dossier EPS n`35, Paris, Editions Revue EPS : 207-227.

FAMOSE J-P, SARRAZIN PH, CURY F (1995) Apprentissage moteur et buts d'accomplissement In : J Bertsch et C Le Scanff. Apprentissage moteur et conditions d'apprentissages Paris, PUF : 89-152.

FONTAYNE P (1999) Motivation et activités physiques et sportives : influence du sexe et du genre sur la pratique du sport et de l'éducation physique. Thèse en Sciences et Techniques des Activités Physiques et Sportives, non-publiée, Université Paris-Sud Orsay.

FONTAYNE P, SARRAZIN P, FAMOSE J-P (sous presse). Les pratiques sportives des adolescents : une différenciation selon le genre. STAPS.

FONTAYNE P, SARRAZIN P, FAMOSE J-P (2000). The Bem Sex-Role Inventory: Validation of a short-version for French teenagers. European Review of Applied Psychology. 50(4) : 405-416.

GRANLEESE J, TREW K, TURNER I (1988) Sex differences in perceived competence. British Journal of Social Psychology. 27 : 181-184.

GUILLET E, SARRAZIN P, FONTAYNE P (2000) « If it is in contradiction with my gender-role, I'll stop! » Introducing survival analysis to study the effects of gender typing on the time of withdrawal from sport practice; a 3-year study. European Review of Applied Psychology. 50(4) : 417-421.

HALL JA, TAYLOR MC (1985) Psychological androgyny and the masculinity-femininity interaction. Journal of Personality and Social Psychology. 49 : 429-435.

HARRY J (1995) Sport ideology, attitudes toward women, and anti-homosexual attitudes. Sex Roles. 32 : 109-116.

HURTIG M-C, PICHEVIN M-F (1985) La variable sexe en psychologie : donné ou construct? Cahiers de Psychologie Cognitive. 5(2): 187-228.

IGNICO AA (1989) Development and verification of gender-role stereotyping index for physical activities. Perceptual and Motor Skills. 68 : 1067-1075.

IGNICO AA, MEAD B (1990) Children's perceptions of the gender appropriateness of physical activities. Perceptual and Motor Skills. 68 : 1067-1075.

JÖRESKOG KG, SÖRBOM D (1993) Lisrel 8: Strucural Equation Modeling with the Simplis command Language. Hillsdale, NJ: Erlbaum.

KOIVULA N (1995) Rating of gender appropriateness of sports participation: Effects of gender based schematic processing. Sex Roles. 33(7/8) : 543-557.

LORENZI-CIOLDI F (1988) Individus dominants et groupes dominés : images masculines et féminines. Grenoble, PUG.

LORENZI-CIOLDI F (1994) Les androgynes. Paris, PUF.

MACCOBY EE (1990) Le sexe, catégorie sociale. Actes de la Recherche en Sciences Sociales. $83: 16-26$.

MARSH HW (1987) Masculinity, femininity and androgyny: Their relations to multiple dimensions of self-concept. Multivariate Behavioral Research. 22 : 91-118.

MARSH HW, BYRNE BM (1991) Differentiated additive androgyny model: Relations between masculinity, femininity and multiple dimensions of self-concept. Journal of Personality and Social Psychology. 61 : 811-828.

MARSH HW, MYERS M (1986) Masculinity, feminity, and androgyny: A methodological and theorical critique. Sex Roles. 14 : 397-430.

MATTEO S (1986) The effect of sex and gender-schematic processing on sport participation. Sex Roles. 15 (7/8) : 417-432. 
MATTEO S (1988) The effect of gender-schematic processing on decisions about sexinappropriate sport behavior. Sex Roles. 18(1/2) : 41-58.

MEAD BJ, IGNICO AA (1992) Children's gender-typed perceptions of physical activity: consequences and implications. Perceptual and Motor Skills. 75 : 1035-1042.

MESSNER MA (1988) Sports and male domination: The female athlete as contested ideological terrain. Sociology of Sport Journal. 5(3) : 197-211.

MESSNER MA (1990) Men studying masculinity: Some epistemological issues in sport sociology. Sociology of Sport Journal. 7: 136-153.

PEDHAZUR EJ, SCHMELKIN LP (1991) Measurement, design, and analysis: An integrated approach. Hillsdale, NJ: Erlbaum.

PELLETT TL, HARRISON JM (1992) Children's perceptions of the gender appropriateness of physical activities: A further analysis. Play and Culture. $5: 305-313$.

PELLETT TL, IGNICO AA (1993) Relationships between children's and parent's stereotyping of physical activities. Perceptual and Motor Skills. 77 : 1283-1289.

RYCKMAN RM, HAMEL J (1995) Male and female adolescents motive related to involvement in organized team sports. International Journal of Sport Psychology. $26: 383-397$.

SALMINEN S (1990) Sex role and participation in traditionally inappropriate sports. Perceptual and Motor Skills. 71(3) : 1216-1218.

SPENCE JT, HALL SK (1996) Children's gender related self-perceptions, activity preferences, and occupationnal stereotypes: A test of three models of gender constructs. Sex Roles. 35 (11/12) : 659-691.

TAP P (1985) Masculin et féminin chez l'enfant. Toulouse, Privat.

TAYLOR MC, HALL JA (1982) Psychological androgyny: Theories, methods and conclusions. Psychological Bulletin. 92:347-366.

VALLERAND RJ (1994) Une introduction à la psychologie sociale contemporaine In : RJ Vallerand. Les fondements de la psychologie sociale, Boucherville (Québec), Gaëtan Morin : 3-52.

VALLERAND RJ, FORTIER MS, GUAY F. (1997) Self-determination and persistence in a Real-Life Setting: Toward a motivational model of high school dropout. Journal of Personality and Social Psychology. 72 : 1161-1176.

WHITLEY BE (1983) Sex role orientation and self esteem: A critical meta analytic review. Journal of Personality and Social Psychology. 44 : 773-786.

WHITLEY BE (1984) Sex role orientation and psychological well-being: Two meta analyses. Sex Roles. 12 : 207-225.

WIGFIELD A, HAROLD RD, FREEDMAN-DOAN C, ECCLES JS, YOON KS, ARBRETON AJA, BLUMENFELD PC (1997) Change in children's competence beliefs and subjective task values across the elementary school years: A 3-year study. Journal of Educational Psychology. 89(3) : 451-469. 